\title{
Long-term training on sand changes lower limb muscle activities during running in runners with over-pronated feet
}

\author{
AmirAli Jafarnezhadgero ${ }^{1 *}$, Amir Fatollahi ${ }^{1}$, Ali Sheykholeslami ${ }^{2}$, Valdeci Carlos Dionisio ${ }^{3}$ and \\ Mohammad Akrami $i^{*^{*}}$ (D)
}

*Correspondence:
amiralijafarnezhad@gmail.
com; m.akrami@exeter.ac.uk
${ }^{1}$ Department
of Sports Management
and Biomechanics, Faculty
of Educational Sciences
and Psychology, University
of Mohaghegh Ardabili,
Ardabil, Iran
${ }^{4}$ Department of Engineering,
University of Exeter,
Exeter EX4 4QF, UK
Full list of author information
is available at the end of the
article

*Correspondence: amiralijafarnezhad@gmail. 1 Department of Sports Management and Biomechanics, Faculty of Educational Sciences

${ }^{4}$ Department of Engineering, University of Exeter,

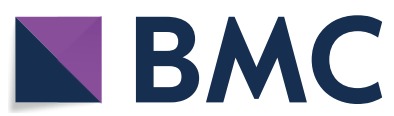

(c) The Author(s), 2021. Open Access This article is licensed under a Creative Commons Attribution 4.0 International License, which permits use, sharing, adaptation, distribution and reproduction in any medium or format, as long as you give appropriate credit to the original author(s) and the source, provide a link to the Creative Commons licence, and indicate if changes were made. The images or other third party material in this article are included in the article's Creative Commons licence, unless indicated otherwise in a credit line to the material. If material is not included in the article's Creative Commons licence and your intended use is not permitted by statutory regulation or exceeds the permitted use, you will need to obtain permission directly from the copyright holder. To view a copy of this licence, visit http:// creativecommons.org/licenses/by/4.0/. The Creative Commons Public Domain Dedication waiver (http://creativecommons.org/publi cdomain/zero/1.0/) applies to the data made available in this article, unless otherwise stated in a credit line to the data.

\begin{abstract}
Background: Running on sand could be a promising exercise intervention for the treatment of over-pronated feet. However, there is a lack of knowledge about the effects of running on sand on muscle activities. Therefore, this study aims to evaluate the long-term effects of running on sand on the activities of selected lower limb muscles in individuals with OPF compared with healthy controls.
\end{abstract}

Methods: Sixty recreational adult male runners with over-pronated feet (foot posture index $>10$ ) were divided into two equal groups (intervention and control). Participants ran barefoot at a pre-defined speed $(\sim 3.3 \mathrm{~m} / \mathrm{s}$ ) over level stable ground both before and after long-term training on the sand. Muscle activities were recorded using a surface bipolar electromyography system.

Results: For the intervention group, we found a reduced foot posture index $(p<0.001$; $d=2.00$ ) and significant group-by-time interactions for gluteus medius activity during the mid-stance phase $(p<0.028 ; d=0.59)$. Significantly higher gluteus medius activity $(p=0.028, d=0.569)$ was found during the post-test. We also observed significant group-by-time interactions for medial gastrocnemius activity during the push-off phase $(p<0.041 ; d=0.54)$. Significantly larger medial gastrocnemius activity $(p=0.041$; $d=0.636)$ was found during the post-test compared to the pre-test.

Conclusions: Long-term running on sand resulted in reduced pronation, increased medial gastrocnemius activity, and improved frontal plane pelvic stability due to higher gluteus medius activity.

Trial registration: IRCT20191211045704N1. Registered 25 February 2020. Retrospectively registered.

Keywords: Flat feet, Lower limb mechanics, Unstable walkway, Electromyography, Running

\section{Background}

Injuries are a major and ongoing inconvenience of running [1]. Incidence rates of running-related injuries (RRIs) can fluctuate from 3 to $85 \%[2,3]$ and 2.5-33 injuries across 1000 h of activity [4], depending on the population studied. About $87 \%$ of RRIs 
occur in the knee, lower leg, foot, and ankle, with abnormal foot posture being a leading cause of those injuries $[5,6]$. The foot is the only part of the body in contact with the ground while running. Therefore, it is responsible for shock absorption and dispersal of ground reaction force across the foot $[7,8]$. An atypical medial foot arch can disrupt the shock absorption and attenuation, imposing more stress on the foot or other structures or joints [9]. Hence, abnormality in the foot has the potential to predispose runners to injuries.

Over-pronated foot (OPF) is the most common functional foot abnormality, with a $2-23 \%$ prevalence rate in adults [10-12]. The deficit in muscular strength, lower limb anatomical alignment, and ligament function have been reported as causes of OPF. It was proposed that the dynamic stabilization of the medial longitudinal arch is reliant on the activity of several muscles, including triceps surae, peroneals, tibialis posterior, and tibialis anterior $[5,8]$. There is evidence that individuals with OPF demonstrate the increased activity of some leg muscles (tibialis posterior, tibialis anterior, toe flexors, medial gastrocnemius, and gluteus medius) and decreased activation of evertor musculature compared to those with normal feet $(5,13,14,15)$. In addition, a previous study reported higher vastus lateralis and vastus medialis muscle activities in healthy controls compared with individuals with PF during drop landing [16]. Accordingly, previous studies $[5,13,16]$ recommended that lower limb muscle activities should be realized during exercise and treatment of individuals with OPF. OPF has been reported as one of the main risk factors predisposing runners to injuries, such as medial tibial stress syndrome, Achilles tendinopathy, and plantar fasciitis [9]. In addition, recently published studies reported that OPF posture is associated with higher odds of RRIs than a normal foot posture $[17,18]$. Therefore, modifying OPF may be a possible way of reducing and preventing RRIs.

Different methods have been utilized in the treatment of OPF. Passive supports such as foot orthoses [19, 20], taping [21, 22, 23], and motion control footwear [24, 25] were suggested in the treatment of OPF. However, therapists often prescribe active exercise interventions and confer additional advantages over passive supports because of the improved foot arches brought about by the strengthening of core foot muscles (intrinsic muscles) [26, 27]. Surprisingly, there is a lack of knowledge about the effects of active interventions on muscle activities in individuals with OPF.

Athletes and coaches have considered sand running an excellent way to complement regular training on a firm surface [28]. From a biomechanical perspective, sand running yields greater net knee extensor activity than stable ground [29]. Examining ground reaction forces (GRFs) and muscle activity while sand walking, Jafarnezhadgero et al. observed that sand walking results in lower peak positive free moments (FM) and loading rate compared with stable ground walking [8]. Furthermore, there is evidence that sand running compared with stable ground running impacts kinematics and kinetics in healthy and diseased individuals $[29,30]$. Therefore, it can be postulated that sand running changes biomechanical factors, muscle activities and modifies OPF [8, 29].

The originality of this study is due to the duration of the training for individuals with OPF. Although Jafarnezhadgero et al. evaluated GRFs and muscle activity in individuals with OPF compared with healthy control ones while sand walking [8], these authors only evaluated the acute effect (and not long term) of walking on sand versus walking on 
a stable surface in individuals with OPF [8]. There is currently a lack of evidence investigating the long-term effects of training on sand and the implications for firm ground performance gains in individuals with OPF. Therefore, we aim to evaluate the long-term effects of sand running on activities of selected lower limb muscles in individuals with OPF. It has been demonstrated that during running on sand, electromyography (EMG) in the gastrocnemius, hamstrings (semimembranosus and biceps femoris), vastii (vastus lateralis and vastus medialis), rectus femoris, and tensor fascia latae were greater than the firm surface measures [29]. Another study indicated that more muscles are mobilized during walking on sand surfaces due to reduced surface elastic energy [31]. Concerning the relevant literature $[8,29,31]$, we hypothesized different lower limb muscle activities after long-term sand running in recreational adult male runners with OPF.

\section{Results}

No significant between-group differences were found at baseline for all the examined variables (Table 1$)$, but the foot posture index was reduced $(p<0.001 ; d=2.00)$ after the running on sand training protocol for the IG (pre: $11.2 \pm 0.7$; post: $9.6 \pm 0.9)$ compared with the CG (pre: $11.0 \pm 0.7$; post: $11.1 \pm 0.7$ ).

Regarding the EMG activity of the selected lower limb muscles during the loading phase, there was no differential effect of "group by time interactions" $(p>0.05$; $d=0.00-0.41)$, nor was there an effect of "time" $(p>0.05 ; d=0.00-0.40)$, or an effect of "group" ( $p>0.05 ; d=0.00-0.41$ ) (Table 2).

For EMG activity of the selected lower limb muscles during the mid-stance phase, there was a significant effect of "group-by-time interactions" for Glut-M activity $(p<0.028 ; d=0.59)$. In the IG, significantly higher Glut-M activity $(p=0.028, d=0.569)$ was found in the post-test compared to the pre-test (Table 3). However, there were no differential effects of "time" ( $p>0.05 ; d=0.00-0.51)$, nor was there an effect of "group" $(p>0.05 ; d=0.00-0.37)$.

For EMG activity of the selected lower limb muscles during the push-off phase, we observed a significant effect of "group-by-time interactions" for Gas-M activity $(p<0.041 ; d=0.54)$ (Table 4$)$. In the IG, significantly greater Gas-M activity ( $p=0.041$; $d=0.636$ ) was found during the post-test compared to the pre-test (Table 4). We also observed that there was no differential effect of "group" ( $p>0.05 ; d=0.00-0.35)$, but there were significant main effects of "time" for Gas-M activity ( $p<0.030 ; d=0.58)$. Pairwise comparisons revealed significantly greater Gas-M activity $(p=0.030 ; d=0.47)$ in the post-test compared with the pre-test (Table 4).

In summary, after the intervention, our results showed increased EMG signals of Glut$\mathrm{M}$ and Gas-M during mid-stance and push-off phases, respectively.

\section{Discussion}

This study aimed to evaluate the long-term effects of sand running on selected lower limb muscle activities in individuals with OPF. The study was the first to evaluate the long-term effects of sand running on active male adults with OPF. Our results highlight sand running as a basis for modifying the foot posture index, Glut-M, and Gas-M activities. The intervention was able to reduce the foot posture index for the IG. We have not measured the EMG activities or strength of intrinsic foot muscles; however, we can 
Table 1 Baseline values of demographic and muscular activity data for all groups

\begin{tabular}{|c|c|c|c|}
\hline Characteristics & Control & Intervention & $p$-value \\
\hline \multicolumn{4}{|l|}{ Parameters } \\
\hline Age (years) & $22.2 \pm 1.9$ & $22.2 \pm 2.5$ & 0.955 \\
\hline Heigh (cm) & $177.9 \pm 5.7$ & $178.0 \pm 6.6$ & 0.869 \\
\hline Mass (kg) & $75.40 \pm 7.9$ & $75.0 \pm 8.2$ & 0.612 \\
\hline Navicular drop (mm) & $16.3 \pm 1.7$ & $16.2 \pm 1.6$ & 0.816 \\
\hline Foot posture index & $11.0 \pm 0.7$ & $11.2 \pm 0.7$ & 0.401 \\
\hline \multicolumn{4}{|l|}{ EMG } \\
\hline \multicolumn{4}{|l|}{ Loading phase } \\
\hline TA & $34.67 \pm 10.23$ & $34.29 \pm 10.91$ & 0.889 \\
\hline Gas-M & $15.20 \pm 3.66$ & $15.48 \pm 4.77$ & 0.795 \\
\hline$V L$ & $15.87 \pm 12.33$ & $15.55 \pm 8.97$ & 0.909 \\
\hline VM & $19.09 \pm 10.08$ & $19.36 \pm 10.28$ & 0.920 \\
\hline RF & $20.38 \pm 7.34$ & $20.79 \pm 10.69$ & 0.865 \\
\hline $\mathrm{BF}$ & $20.94 \pm 11.94$ & $25.61 \pm 11.81$ & 0.133 \\
\hline ST & $15.96 \pm 7.03$ & $15.55 \pm 5.97$ & 0.807 \\
\hline Glut-M & $31.01 \pm 14.34$ & $31.36 \pm 11.30$ & 0.915 \\
\hline \multicolumn{4}{|l|}{ Mid stance phase } \\
\hline TA & $25.95 \pm 11.10$ & $26.26 \pm 11.75$ & 0.917 \\
\hline Gas-M & $51.09 \pm 16.55$ & $51.98 \pm 17.47$ & 0.840 \\
\hline$V L$ & $46.50 \pm 18.53$ & $46.84 \pm 18.91$ & 0.945 \\
\hline VM & $55.70 \pm 26.89$ & $53.56 \pm 21.21$ & 0.733 \\
\hline RF & $36.51 \pm 13.52$ & $37.88 \pm 16.51$ & 0.726 \\
\hline $\mathrm{BF}$ & $23.93 \pm 13.63$ & $24.13 \pm 11.25$ & 0.951 \\
\hline ST & $20.18 \pm 9.28$ & $21.50 \pm 8.09$ & 0.561 \\
\hline Glut-M & $42.06 \pm 14.79$ & $42.15 \pm 12.38$ & 0.981 \\
\hline \multicolumn{4}{|l|}{ Push off } \\
\hline TA & $17.13 \pm 9.14$ & $17.17 \pm 7.69$ & 0.986 \\
\hline Gas-M & $54.85 \pm 16.04$ & $54.50 \pm 16.95$ & 0.933 \\
\hline$V L$ & $25.73 \pm 8.59$ & $26.45 \pm 9.88$ & 0.764 \\
\hline VM & $19.64 \pm 5.77$ & $20.07 \pm 6.41$ & 0.785 \\
\hline RF & $20.35 \pm 5.77$ & $20.92 \pm 5.37$ & 0.695 \\
\hline $\mathrm{BF}$ & $19.14 \pm 7.93$ & $19.98 \pm 7.28$ & 0.671 \\
\hline ST & $11.84 \pm 5.54$ & $11.72 \pm 4.73$ & 0.927 \\
\hline Glut-M & $24.59 \pm 14.12$ & $24.57 \pm 11.74$ & 0.995 \\
\hline \multicolumn{4}{|l|}{ Swing phase } \\
\hline TA & $29.31 \pm 11.42$ & $28.67 \pm 14.65$ & 0.851 \\
\hline Gas-M & $11.26 \pm 5.05$ & $11.27 \pm 4.49$ & 0.996 \\
\hline$V L$ & $20.59 \pm 9.43$ & $20.58 \pm 8.90$ & 0.997 \\
\hline VM & $23.50 \pm 10.68$ & $23.45 \pm 9.53$ & 0.986 \\
\hline RF & $19.85 \pm 7.28$ & $19.99 \pm 5.81$ & 0.935 \\
\hline $\mathrm{BF}$ & $14.60 \pm 8.91$ & $13.27 \pm 6.43$ & 0.512 \\
\hline ST & $14.39 \pm 7.81$ & $14.11 \pm 7.57$ & 0.887 \\
\hline Glut-M & $23.35 \pm 8.73$ & $22.81 \pm 8.22$ & 0.807 \\
\hline
\end{tabular}

EMG electromyography, $T A$ tibialis anterior, Gas- $M$ gastrocnemius medialis, $B F$ biceps femoris; $S T$, semitendinosus, $V L$ vastus lateralis, $V M$ vastus medialis, $R F$ rectus femoris, Glut $M$ M gluteus medius; P-value from independent samples t-test, $S D$ standard deviation 


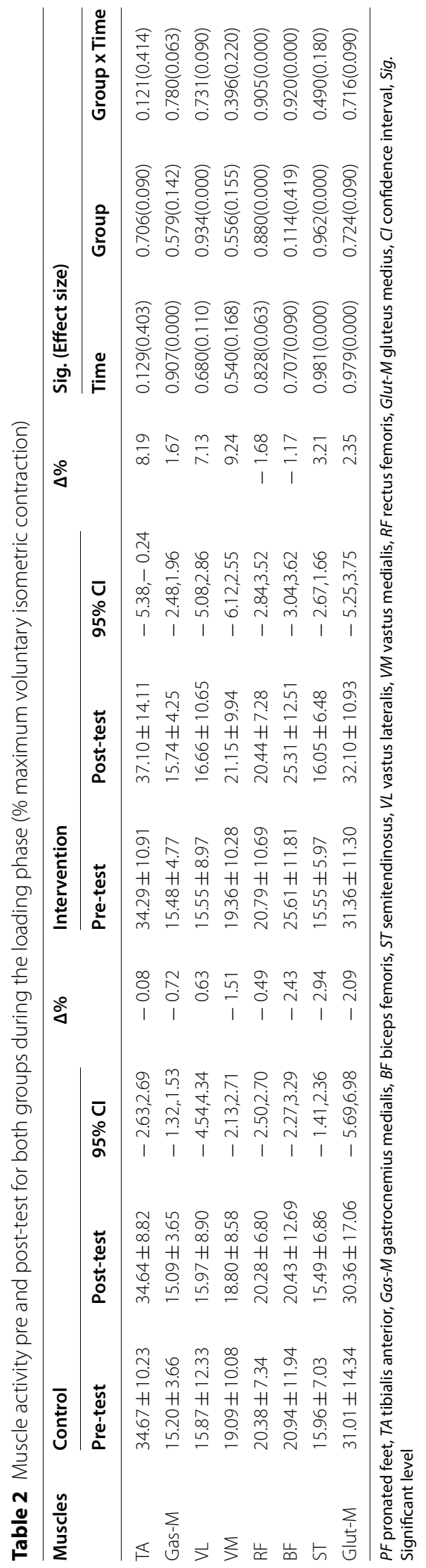




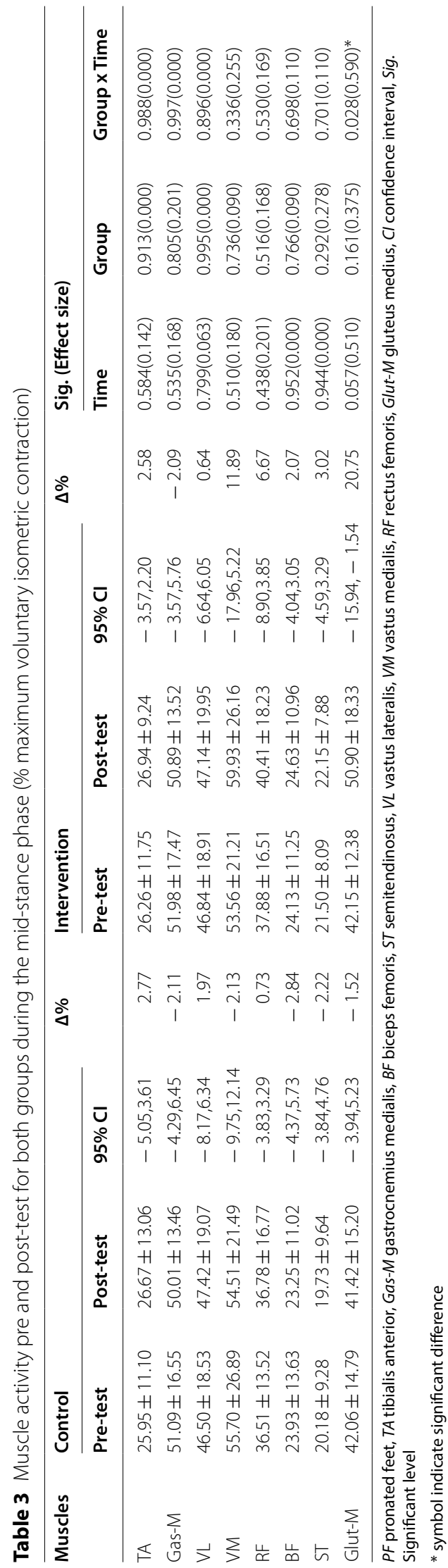




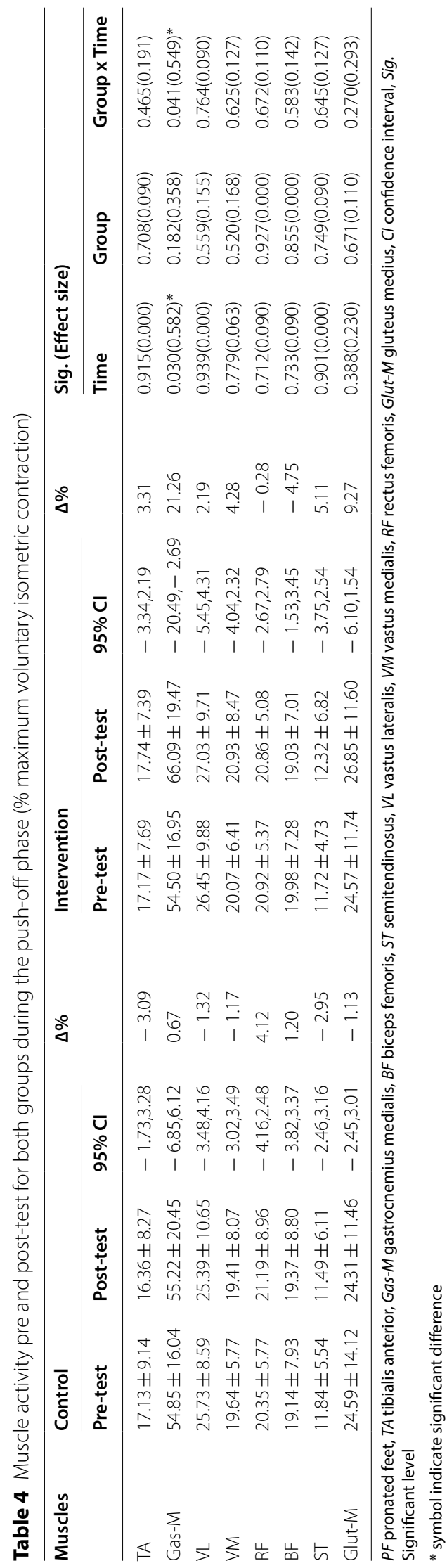


assume that the intrinsic foot muscles were strengthened. In a pronated foot, abnormal alignment may stretch and weaken the intrinsic foot muscles by elongating them beyond their neutral physiological resting position. In addition, the alignment changes the length-tension relationship of the muscles, which may preclude the muscle from generating sufficient or optimal force. Various methods have been advocated in treating a pronated foot, including active strengthening exercises [32, 33, 34]. Several active exercises can be used to strengthen the intrinsic foot muscles, reduce foot pronation, and raise the medial longitudinal arch, such as picking up objects, engaging in unilateral balance activities, and performing shin curls, towel toe curls, and the short foot exercise [32, 34].

Short foot exercise is frequently prescribed and performed in sports and rehabilitation to strengthen the intrinsic foot muscles and enhance the longitudinal and transverse arches. A study reported that the short foot exercise (e.g., sand training) was more effective than towel toe curls in activating the abductor hallucis muscles and preventing a lowered medial longitudinal arch [26]. A previous study also observed that the intrinsic strength of the foot increased as the foot posture index was reduced [35]. The improved strength of the intrinsic muscles might also improve the energy transfer across the lower limb [36]. The training program includes six exercises. From our study design, it is unclear exactly which exercise affected EMG and foot posture index changes. Further study is warranted to better established this issue.

Our results revealed greater Glut-M activities during the mid-stance phase after longterm sand running (IG). Previous studies have shown that during the early phase of stance, the knee valgus is associated with hip adduction in individuals with OPF. This condition causes greater hip abductor activities, mainly due to the greater activity of the Glut-M muscle [37]. In addition, the weakness of the Glut-M muscles may increase the risk of sustaining injuries attributed to excessive subtalar pronation [11]. During running, the Glut-M muscle contracts to maintain lower limb alignment from the pelvis to the foot $[38,39]$. However, the foot's intrinsic muscle function is also critical in the mid-stance phase to avoid excessive pronation. The increased Glut-M muscle activity could be associated with reduced pronation, leading to new lower limb alignment and Glut-M muscle activation. Following our results and the previous study, we propose that sand running could be an effective rehabilitative means to treat lower limb injuries due to producing greater muscle activities [40].

The IG also showed significantly greater Gas-M activity at post-test than pre-test. To the authors' knowledge, no study has examined the effects of long-term sand running on muscular activities in OPF individuals. However, a previous study showed that running on sand significantly increased the calf circumference over the training period. This condition may indicate a greater overload stimulus in that particular muscle group [41]. In addition, supporting our results, Pinnington et al. identified a significantly greater peak activation of the gastrocnemius when running on sand versus grass, primarily during the push-off phase of running, where there is plantar flexion of the foot [29]. Another previous investigation [42] reported that excessive rear-foot eversion during the stance phase of gait might result in increased internal rotation of the tibia with respect to the talus; associated joint coupling would cause the hip to internally rotate to a greater degree, thereby also increasing hip adduction and the dynamic Q angle [42]. Therefore, increasing the activity of Glut-M along with gastrocnemius may be increased the hip abduction 
and decrease the rear-foot eversion, respectively. Therefore, training on a continuously unstable bearing surface such as sand can improve the running mechanics by utilizing diverse muscle groups and increasing joint mobility. These results are consistent with a previous study indicating that athletic training on sand surfaces improved strength and endurance in the calf and thigh muscles [41].

Certain limitations of the present study must be acknowledged. First, the intervention group performed the exercise training on the sand, while the control group did not perform any exercise. This design cannot clarify which factor contributes to the changes in the EMG and foot posture index. That is, these changes might be due to just the (1) the intervened exercise irrespective of the ground surface (i.e., the observed training effect is due to the exercise type or not), (2) touching the sand irrespective of physical activities (i.e., surface-specific training effect or not), or (3) barefoot exercise itself irrespective of the ground surface or exercise types (i.e., barefoot-specific training effect or not). Second, we did not assess healthy control individuals and we could not say that the same protocols should be performed with the non-OPF runners. Therefore, further studies with different control groups are warranted to evaluate better the effects of exercise training on the sand on running biomechanics and muscle activities. Third, we examined the long-term effects of sand running only for active male individuals with OPF. Therefore, our results cannot be generalized to active female individuals. Future studies are needed to examine the long-term effects of sand running in females with OPF to establish whether sand is a preventive/rehabilitative means of reducing static foot pronation and improving muscle activities. Fourth, we did not examine running kinematics in the present study. Therefore, it is recommended for future studies to investigate the effect of sand running on kinematics and dynamic foot posture during running. Running kinematic assessment could reveal adaptations such as increased or decreased foot pronation and knee flexion to long-term sand running. Concerning the training studies on the sand to date, there is still a need for further research to determine the full range of physiological and biomechanical benefits associated with sand. The generalization should be considered carefully for the reasons described above.

\section{Conclusions}

Long-term running on sand resulted in reduced pronation and increased Gas-M activity and improved frontal plane pelvic stability due to greater Glut-M activity in individuals with OPF. These findings can be attributed to the fact that training on sand requires more diverse muscles in individuals with OPF. Hence, we can confirm the potential of sand as a new training ground material when attempting to improve the walking ability, particularly the running mechanics in individuals with OPF. In addition, the increased hip and knee range of running on sand can be partly attributed to the increased EMG activation of the Gas-M and Glut-M muscles in individuals with OPF [7]. Although a complete evaluation and studies are necessary, our results can provide insights for researchers and clinicians to prevent or treat injuries in individuals with OPF, especially when dealing with RRIs in these individuals. 


\section{Methods}

\section{Participants}

We used the G*Power and data in a previous study examining running muscle activities in individuals with OPF. The calculation parameters were power analysis of 0.05 (type I error) and 0.20 (type error II), i.e., $80 \%$ statistical power. Also considered were two tests (pre, post), the correlation coefficient of 0.5 , and the effect size of 0.80 for running muscle activities (i.e., maximal tibialis anterior activity) [8]. As a result, 30 participants would be necessary to observe large group-by-time interactions.

In November 2019, individuals were recruited from physical therapy clinics in Ardebil city, Iran. An orthopedic surgeon assessed all individuals before selection. The eligibility criteria included a navicular drop of more than $10 \mathrm{~mm}$ [43], a foot posture index of $>10$ [44], and a rearfoot striker. In the current study, a modified version of the navicular drop described by Brody [45] was used to determine the sagittal plane displacement of the navicular between the resting (seated) and stand on one leg positions. The participant was seated with both feet flat on the ground and knees flexed at $90^{\circ}$. The most medial aspect of the navicular was marked. A blank card was held at right angles to the foot against the navicular marking with the base of the card flat on the supporting surface. The height of the navicular was marked on the card. Then, the participant was asked to stand on one leg by flexing the contralateral knee. The singlelimb stance position was selected, because recent work by McPoil and Cornwall [46] has shown that measurements taken from this position more accurately represent the position of the foot during the midstance phase of gait. A blank card was held at right angles to the foot against the navicular marking with the base of the card flat on the supporting surface. The height of the navicular was marked on the card. The difference between the height of the navicular in the resting (seated) and stand on one leg positions was recorded as the navicular drop. The foot posture index consists of six items to quantify and classify foot posture [44, 47]. These are (i) palpation of the head of the talus; (ii) curvatures above and below the lateral malleolus; (iii) position of the calcaneus in the frontal plane; (iv) prominence of the malleolus; (v) congruence of the medial longitudinal arch; and (vi) abduction/adduction of the forefoot. Each item was rated on a visual analog scale ranging from -2 to +2 , resulting in a total score of -12 to +12 . Negative values indicate supinated foot posture, and positive values indicate pronated foot posture. Of note, values of 10-12 in the foot posture index were classified as over-pronated feet $[44,47]$. The foot posture index was evaluated by a podiatrist with $\sim 10$ years of professional experience. The validity of the foot posture index has been investigated fully and reported previously [44]. The foot posture index predicted $64 \%$ of the variance in static standing posture and $41 \%$ of the variance in the mid-stance posture during normal walking and demonstrated good inter item reliability (Cronbach's $\alpha=0.83$ ) [44]. A more recent study has also demonstrated good internal construct validity and fit of the scoring system with Rasch model, a useful statistical model of the uni-dimensionality (capacity to measure a single construct) and scale stability (or linearity across a range of values) of a measure [48]. A detailed description of the foot posture index can be found elsewhere [44, 47]. In addition, the eligibility criteria were right-footed and physically active individuals with at least 
1 year of experience of recreational running training with three sessions per week. The volunteers should also use the rearfoot strike to land the foot on the ground.

Individuals were removed from the study if they had limb length discrepancies of more than $5 \mathrm{~mm}$, or reported muscle spasm, neuromuscular disorders, orthopedicrelated diseases, or any previous surgery in the lower limbs and trunk. Only males were recruited for the present study, as previous studies showed distinct biomechanical characteristics between females and males $[49,50]$. Females were associated with significantly greater knee abduction, knee internal rotation, and ankle eversion, while males were associated with substantially greater hip flexion [51]. Ferber et al. examined the gender differences in 3-D kinematics of the hip and knee. Female runners exhibited greater peak hip adduction, hip internal rotation, and knee abduction than men [52].

Eighty-five recreational, right-footed male runners with OPF were assessed; 25 were excluded (21 did not meet the eligibility criteria, two refused to participate in the study, and two for other reasons) (Fig. 1). Thus, 60 recreational male runners with OPF were randomly allocated to the intervention group (IG) ( $n=30$; age: $22.2 \pm 2.5$ years; height: $178.0 \pm 6.6 \mathrm{~cm}$; mass: $75.0 \pm 8.2 \mathrm{~kg})$ and control group (CG) $(n=30$; age: $22.2 \pm 1.9$ years; height: $177.9 \pm 5.7 \mathrm{~cm}$; mass: $75.4 \pm 7.9 \mathrm{~kg}$ ).

The participants were randomized into the IG and CG. In addition, during the randomization process, a set of sealed, opaque envelopes was used to ensure the concealment of allocation. Thus, those evaluating the participants were unaware of the group allocation (i.e., blind assessors). All participants had their dominant foot ascertained

\section{CONSORT 2010 Flow Diagram}

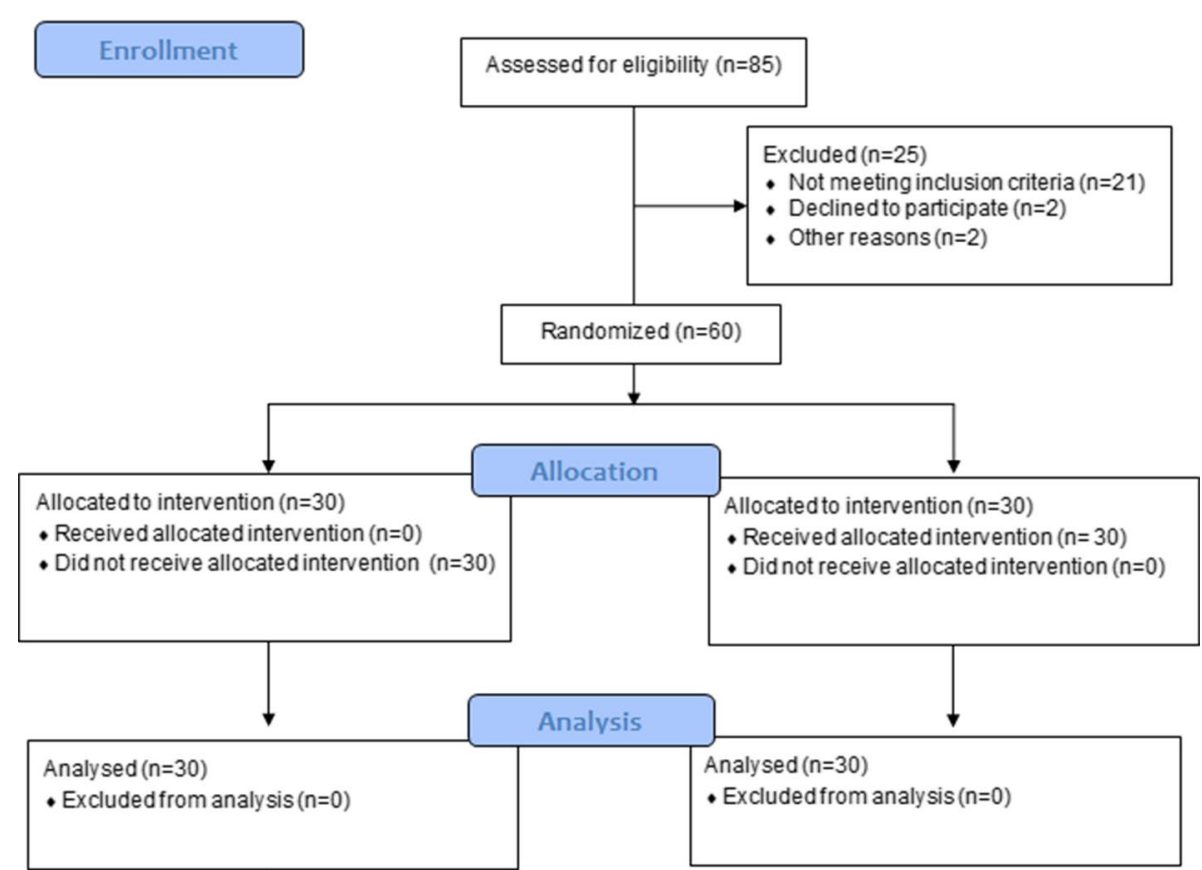

Fig. 1 Flow diagram of the double-blinded randomized controlled trial 
using a kicking ball test and received an explanation of the procedures before obtaining informed consent.

\section{Experimental procedures}

The participants performed a warm-up protocol including stretching the lower limb muscles and $5 \mathrm{~min}$ of self-selected running speed to familiarize themselves with the laboratory environment [53]. Next, all participants ran barefoot over an $18 \mathrm{~m}$ runway. Two sets of infrared photocells positioned $6 \mathrm{~m}$ apart along the length of the runway were used to monitor the running speed and set it at a speed of $3.3 \mathrm{~m} / \mathrm{s} \pm 5 \%$ [54]. The photocells were placed at approximately shoulder height to avoid being triggered by arm swing [55]. Running at this speed has previously been used for determining running-related risk factors of injuries [56]. A trial was considered successful if the dominant foot (the dominant limb was measured) landed in the force plate center and if the EMG signals were clean upon visual examination. Five successful running trials, both pre-test and post-test, were used for data analysis [29]. Maximum voluntary isometric contraction (MVIC) tests were then applied for each muscle to normalize the EMG data (Appendix $1)$.

\section{Experimental setup and data processing}

A force plate (Bertec Corporation, Columbus, OH, USA) was located at the center of the level stable runway. A force plate with a sampling rate of $1000 \mathrm{~Hz}$ was used to register the GRF data. The description of the kinetic data process can be found in detail in a previous study [57]. The GRF data was processed using a low-pass filter at $20 \mathrm{~Hz}$ (4th order Butterworth filter, zero lag). The threshold of $10 \mathrm{~N}$ was used to determine the heel strike and toe-off.

Eight pairs of bipolar $\mathrm{Ag} / \mathrm{AgCl}$ surface electrodes $(25 \mathrm{~mm}$ center-to-center distance; input impedance of $100 \mathrm{M} \Omega$; and common-mode rejection ratio of $>110 \mathrm{~dB}$ ) were used to register the muscle activities of tibialis anterior (TA), gastrocnemius medialis (Gas$\mathrm{M})$, biceps femoris $(\mathrm{BF})$, semitendinosus $(\mathrm{ST})$, vastus lateralis $(\mathrm{VL})$, vastus medialis (VM), rectus femoris (RF), and gluteus medius (Glut-M) of the dominant leg [8]. The raw EMG signals were digitized at $1000 \mathrm{~Hz}$ using a wireless EMG system (Biometrics Ltd, Nine Mile Point Ind. Est., Newport, UK). Before electrode fixation, the skin was gently shaved, rubbed, and cleaned with alcohol. The Nexus software (Oxford Metrics, Oxford, UK) synchronized the GRF and EMG data, which was processed according to a previous study [37]. The run was divided into three phases to analyze the EMG data: the loading (0-20\% stance phase), mid-stance (20-50\% stance phase), and push-off (50-100\% stance phase) phases [58, 59, 60] (Fig. 2). All raw EMG data were processed in a custom program written in MATLAB (Release 12, MathWorks Inc.). First, raw EMG data were high-pass filtered using a zero-lag fourth-order recursive Butterworth filter (cutoff frequency $10 \mathrm{~Hz}$ ) to remove movement artifacts. Then, full-wave was rectified and filtered using a low pass Butterworth filter (cutoff frequency of $6 \mathrm{~Hz}$ ). The muscle activation profile was then normalized to the MVIC value for each respective subject. The aligned EMG data were then normalized to the stance (30 data points) and stride (51 data points), interpolated using a cubic spline, and then exported to Microsoft Excel. 


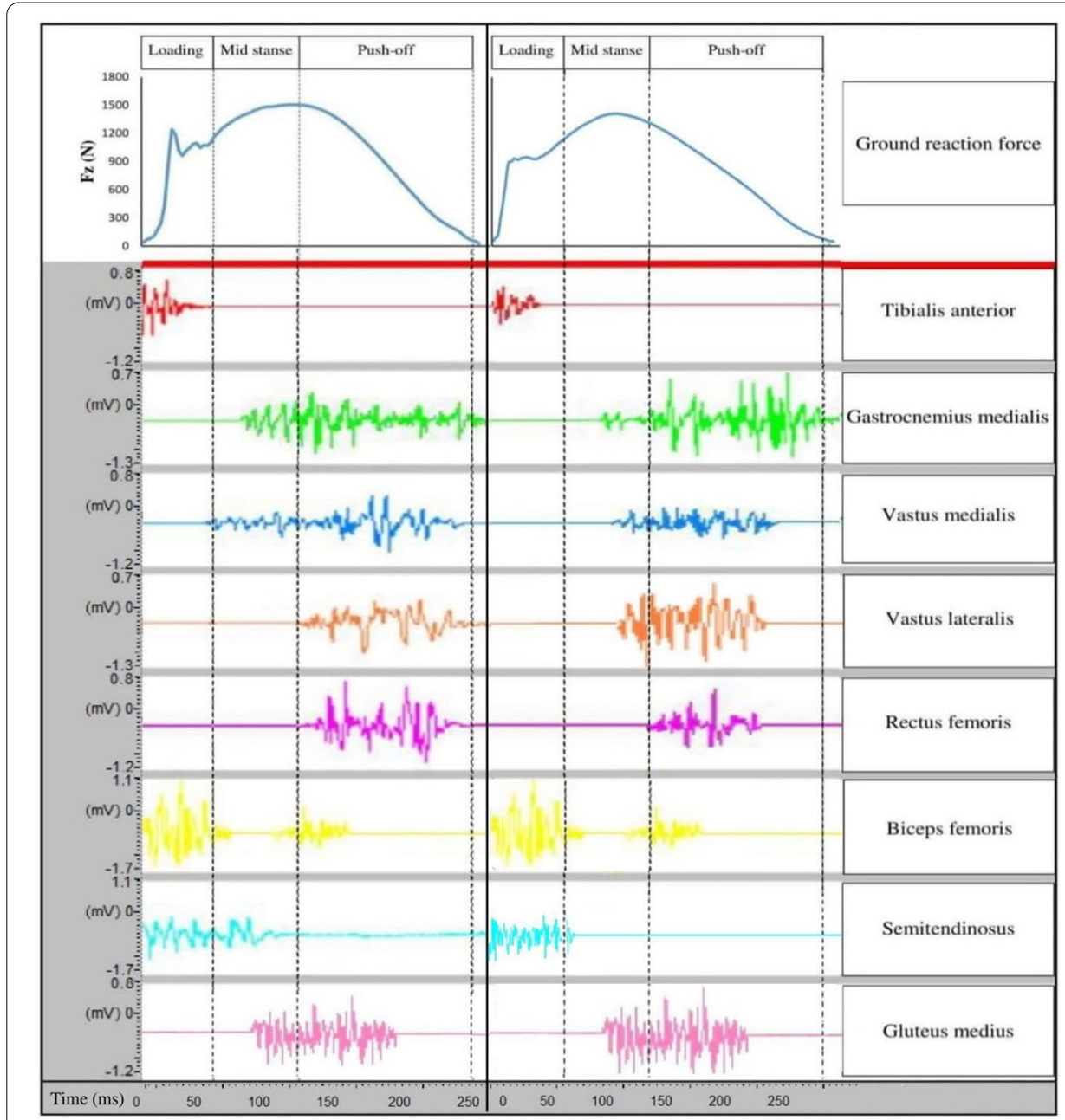

Fig. 2 Loading, mid stance, and push-off phase of running during both pre-test (left side) and post-test (right side). Fz stand for vertical ground reaction force.

The following EMG parameters were then extracted for analysis: EMG of individual muscles expressed as a fraction of MVIC over the running phases [29].

\section{Sand running training protocol}

The IG performed the sand running training program, including continuous jogging, striding, bounding, galloping, and short sprints, for 8 weeks (three sessions per week) $[53,61]$. The training program was carried out in barefoot conditions. Each session was started with a warm-up and stretching session for 5 min and ended with a 5-min warm-down session [53]. Training duration was 50 min per session [53] (Table 5; Fig. 3). Participants started the stride by running at low speed, focusing on a short, quick stride for striding. After that, they gradually increased their speed using longer strides. During the exercise, individuals were asked to keep their torso extended and relaxed. For bounding, a straight-leg bound exercise was used to develop the power output of the lower limbs. For this purpose, participants started the exercise with their feet hip-width apart. After that, they started the first bound by sweeping the 


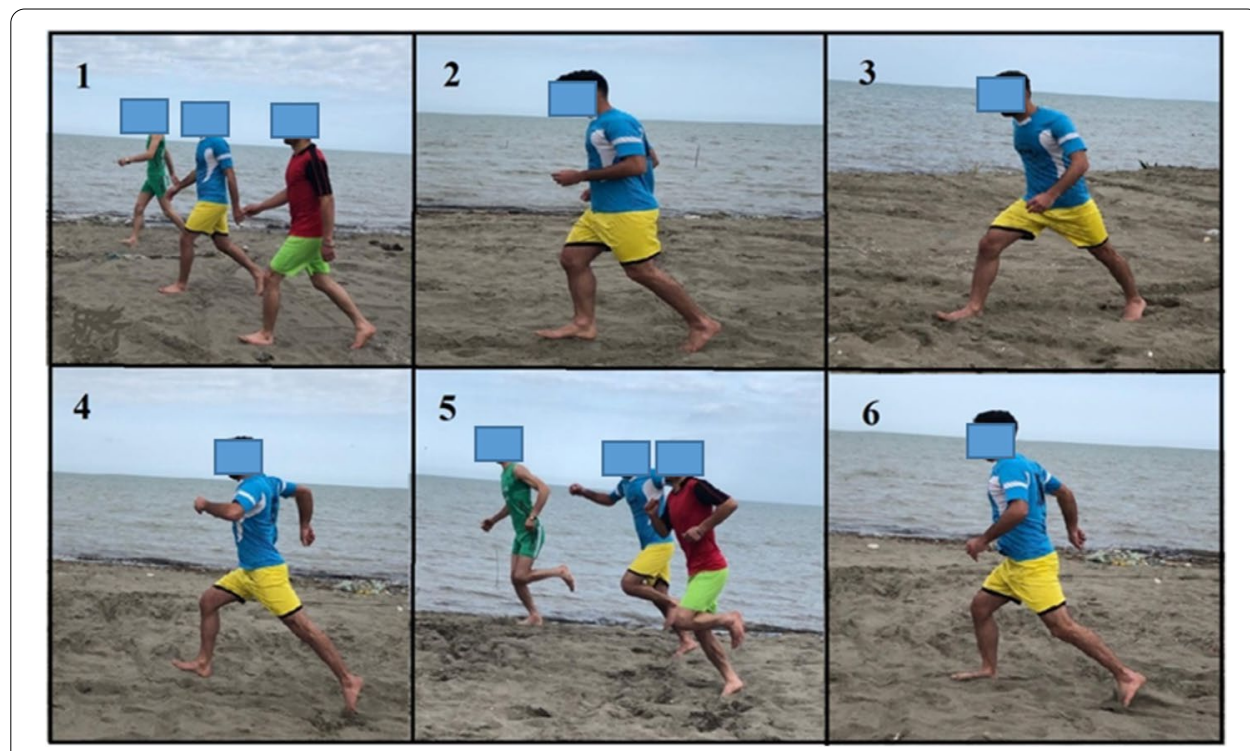

Fig. 3 Examples taken from the progressively designed exercise program on sand. $\mathbf{1}$ walking exercise; $\mathbf{2}$ jogging exercise; $\mathbf{3}$ striding exercise; $\mathbf{4}$ bounding exercise; $\mathbf{5}$ galloping exercise; and $\mathbf{6}$ short sprints

Table 5 Exercise protocol of the intervention group

\begin{tabular}{lclll}
\hline Exercise & $\begin{array}{l}\text { Duration } \\
\text { (minutes) }\end{array}$ & $\begin{array}{l}\text { Repetitions } \\
\text { (number) }\end{array}$ & Distance (meters) & $\begin{array}{l}\text { Recovery } \\
\text { period } \\
\text { (minutes) }\end{array}$ \\
\hline Walking & 5 & - & 50 & - \\
Continuous jogging & 20 & - & 50 & - \\
Striding & 3 & $2-3$ & 50 & 1 \\
Bounding & 3 & $2-3$ & 30 & 1 \\
Galloping & 3 & $2-3$ & 30 & 1 \\
Short sprints & 6 & $3-5$ & 25 & 2 \\
\hline
\end{tabular}

lead leg forward with the knee joint fully extended. At the same time, the opposite arm swept forward to match the range of the lead leg. During the following movement sequence, participants quickly pulled the lead leg back toward the ground with the foot in dorsiflexed position to prepare for a dynamic landing. During the exercise, participants were asked to realize midfoot ground contact with fully extended posture. The knee was extended during landing. Thereafter, participants moved the knee of the free leg forward to initiate the second straight-leg bound. They performed this cyclical bounding movement for a distance of $30 \mathrm{~m}$. Galloping was performed with either foot as the lead. For this purpose, one foot was placed in front of the opposite foot. The front foot took a large step forward, while the second foot remained in place. After that, the back foot took a step forward but always remained behind the front foot. The $25 \mathrm{~m}$ sprints began with the participants in a forward lunge position. Time was started on the command "go" and stopped when the individual's foot touched the finish line. The elapsed time was measured using a handheld watch with an accuracy 
of $0.1 \mathrm{~s}$. Participants were instructed to perform the test at maximal effort and as fast as possible.

A physiotherapist supervised each session to modify the exercise or the progression to meet the given training program and ensure the correct technique. We evaluated the IG after the intervention, scheduled 6 days after the final session. We used this procedure to avoid interference of acute physiological responses to training [62]. We also evaluated CG for the second time after 9 weeks, but the group participants did not receive any exercise. Individuals from the CG followed their regular daily routine and did not perform additional exercise during the intervention period. After the intervention period, individuals from the CG could receive the same exercise program as the IG. All participants were asked not to take up any extra physical activity or exercise during the experimental period. Table 5 illustrates the training exercises for the IG [53].

\section{Statistical analyses}

The Shapiro-Wilk test evaluated and confirmed the data normality, and the data were presented in mean and standard deviations. A mixed ANOVA (time: pre vs. post) $\times$ (group: CG vs. IG) was used to compare outcomes between groups over time. Post-hoc analyses were calculated using the Bonferroni test. We determined the effect sizes by converting partial eta-squared $(\eta 2 \mathrm{p})$ to Cohen's $\mathrm{d}(d<0.50$ indicate small effects, $0.50 \leq d<0.80$ indicate medium effects, and $d \geq 0.80$ indicate large effects). All analyses were performed using SPSS version 24.0, with a significance level set at $p<0.05$.

\section{Appendix 1}

Description of the maximum voluntary isometric contraction (MVIC) tests for tibialis anterior (TA), gastrocnemius medialis (Gas-M), biceps femoris (BF), semitendinosus $(\mathrm{ST})$, vastus lateralis $(\mathrm{VL})$, vastus medialis $(\mathrm{VM})$, rectus femoris $(\mathrm{RF})$, and gluteus medius (Glut-M) muscles.

\begin{tabular}{ll}
\hline Muscles & Test protocol \\
\hline TA & $\begin{array}{l}\text { In seated position on a chair with back rest, with } 90^{\circ} \text { hip, knee, and ankle joint flexion. Participants } \\
\text { were asked to activate TA at maximal effort against resistance }\end{array}$ \\
Gas-M $\quad \begin{array}{l}\text { In seated position on the examination table with the hip flexed at } 90^{\circ} \text { and the knee and ankle in neu- } \\
\text { tral position. Participants activated their plantar flexors at maximal effort against resistance }\end{array}$ \\
BF & $\begin{array}{l}\text { In seated position on a chair with hip and knee flexed at } 90^{\circ} \text {. Participants activated the hamstring } \\
\text { muscles at maximal effort against resistance }\end{array}$ \\
ST & $\begin{array}{l}\text { In seating position on a chair with hip and knee flexed at } 90^{\circ} \text {. Participants maximally activated their } \\
\text { knee flexors against resistance } \\
\text { In seated position on a chair with hip and knee flexed at } 90^{\circ} \text {. Participants maximally activated their } \\
\text { knee extensors against resistance }\end{array}$ \\
\hline
\end{tabular}

\section{Abbreviations}

RRIs: Running-related injuries; OPF: Over-pronated foot; GRFs: Ground reaction forces; FM: Free moment; EMG: Electromyography; IG: Intervention group; CG: Control group; MVIC: Maximum voluntary isometric contraction; TA: Tibialis anterior; Gas-M: Gastrocnemius medialis; BF: Biceps femoris; ST: Semitendinosus; VL: Vastus lateralis; VM: Vastus medialis; RF: Rectus femoris; Glut-M: Gluteus medius. 


\section{Acknowledgements}

The authors would like to acknowledge the Ardabil University of Medical Sciences, Ardabil, Iran, for supporting this project.

\section{Authors' contributions}

The authors have equal contributions in preparing this manuscript. All authors read and approved the final manuscript.

\section{Funding}

None.

\section{Availability of data and materials}

The data sets used and/or analyzed during the current study are available from the corresponding author on reasonable request.

\section{Declarations}

\section{Ethics approval and consent to participate}

A double-blind, randomized controlled trial protocol was approved by the ethical committee of the Ardabil University of Medical Sciences, Ardabil, Iran (IR.ARUMS.REC.1397.091), with the Trial Registration Number also this study registered in the Iranian Registry of Clinical Trials (IRCT20191211045704N1). Informed consent to participate in the study was obtained from participants, and their rights were protected throughout the study. Name of the registry: Iranian Registry of Clinical Trials. URL of the trial registry record: https://fa.irct.ir/user/trial/44427/view. Date of enrolment of the first participant to the trial: 12/10/2019. Date of Tria's registration: 02/25/2020. Was your trial registered before the first participant was enrolled? No.

\section{Consent for publication}

Informed consent to participate in the study was obtained from participants, and their rights were protected throughout the study, which the ethical committee approved of the Ardabil University of Medical Sciences, Ardabil, Iran.

\section{Competing interests}

The authors report no conflict of interests.

\section{Author details}

${ }^{1}$ Department of Sports Management and Biomechanics, Faculty of Educational Sciences and Psychology, University of Mohaghegh Ardabili, Ardabil, Iran. ${ }^{2}$ Department of Counseling, Faculty of Educational Sciences and Psychology, University of Mohaghegh Ardabili, Ardabil, Iran. ${ }^{3}$ Physical Education and Physiotherapy Faculty, Federal University of Uberlândia, Uberlândia, Brazil. ${ }^{4}$ Department of Engineering, University of Exeter, Exeter EX4 4QF, UK.

Received: 2 August 2021 Accepted: 10 November 2021

Published online: 27 November 2021

\section{References}

1. Ceyssens L, Vanelderen R, Barton C, Malliaras P, Dingenen B. Biomechanical risk factors associated with runningrelated injuries: a systematic review. Sports Med. 2019;49(7):1095-115.

2. Kluitenberg B, van Middelkoop M, Diercks R, van der Worp H. What are the differences in injury proportions between different populations of runners? A systematic review and meta-analysis. Sports Med. 2015;45(8):1143-61.

3. Van Gent R, Siem D, van Middelkoop M, Van Os A, Bierma-Zeinstra S, Koes B. Incidence and determinants of lower extremity running injuries in long distance runners: a systematic review. Br J Sports Med. 2007;41(8):469-80.

4. Videbæk S, Bueno AM, Nielsen RO, Rasmussen S. Incidence of running-related injuries per $1000 \mathrm{~h}$ of running in different types of runners: a systematic review and meta-analysis. Sports Med. 2015;45(7):1017-26.

5. Murley GS, Landorf KB, Menz HB, Bird AR. Effect of foot posture, foot orthoses and footwear on lower limb muscle activity during walking and running: a systematic review. Gait Posture. 2009;29(2):172-87.

6. Williams DS III, Davis IM, Scholz JP, Hamill J, Buchanan TS. High-arched runners exhibit increased leg stiffness compared to low-arched runners. Gait Posture. 2004;19(3):263-9.

7. Winter DA. Biomechanics and motor control of human movement. Wiley; 2009.

8. Jafarnezhadgero A, Fatollahi A, Amirzadeh N, Siahkouhian M, Granacher U. Ground reaction forces and muscle activity while walking on sand versus stable ground in individuals with pronated feet compared with healthy controls. PLoS ONE. 2019;14(9):e0223219.

9. Benca E, Ziai P, von Skrbensky G, Ahmetović A, Windhager R, Krpo S. Running injuries. Medicinski Zurnal. 2013;19(4):345-53.

10. Aenumulapalli A, Kulkarni MM, Gandotra AR. Prevalence of flexible flat foot in adults: a cross-sectional study. J Clin Diagn Res JCDR. 2017;11(6):17.

11. Ukoha U, Egwu O, Okafor I, Ogugua P, Igwenagu V. Pes planus: incidence among an adult population in Anambra State, Southeast Nigeria. Int J Biomed Adv Res. 2012;3(3):166-8.

12. Bhoir T, Anap DB, Diwate A. Prevalence of flat foot among 18-25 years old physiotherapy students: cross sectional study. Indian J Basic Appl Med Res. 2014;3(4):272-8.

13. Hunt AE, Smith RM. Mechanics and control of the flat versus normal foot during the stance phase of walking. Clin Biomech. 2004;19(4):391-7. 
14. Gray EG, Basmajian JV. Electromyography and cinematography of leg and foot ("normal" and flat) during walking. Anat Rec. 1968;161(1):1-15.

15. Khodaveisi H, Sadeghi H, Memar R, Anbarian M. Comparison of selected muscular activity of trunk and lower extremities in young women's walking on supinated, pronated and normal foot. Apunts Medicina de l'Esport. 2016;51(189):13-9.

16. Chang JS. Comparisons of vastus medialis and vastus lateralis muscle activities according to different heights during drop landing in flatfooted adults. J Korean Phys Ther. 2020;32(5):302-6.

17. Hein T, Janssen P, Wagner-Fritz U, Haupt G, Grau S. Prospective analysis of intrinsic and extrinsic risk factors on the development of A chilles tendon pain in runners. Scand J Med Sci Sports. 2014;24(3):e201-12.

18. Winkelmann ZK, Anderson D, Games KE, Eberman LE. Risk factors for medial tibial stress syndrome in active individuals: an evidence-based review. J Athl Train. 2016:51(12):1049-52.

19. Alavi-Mehr SM, Jafarnezhadgero A, Salari-Esker F, Zago M. Acute effect of foot orthoses on frequency domain of ground reaction forces in male children with flexible flatfeet during walking. Foot. 2018;37:77-84.

20. Jafarnezhadgero A, Madadi-Shad M, Alavi-Mehr SM, Granacher U. The long-term use of foot orthoses affects walking kinematics and kinetics of children with flexible flat feet: a randomized controlled trial. PLoS ONE. 2018;13(10):e0205187.

21. Aguilar MB, Abián-Vicén J, Halstead J, Gijon-Nogueron G. Effectiveness of neuromuscular taping on pronated foot posture and walking plantar pressures in amateur runners. J Sci Med Sport. 2016;19(4):348-53.

22. Cheung RT, Chung RC, Ng GY. Efficacies of different external controls for excessive foot pronation: a meta-analysis. Br J Sports Med. 2011;45(9):743-51

23. Luque-Suarez A, Gijon-Nogueron G, Baron-Lopez FJ, Labajos-Manzanares MT, Hush J, Hancock MJ. Effects of kinesiotaping on foot posture in participants with pronated foot: a quasi-randomised, double-blind study. Physiotherapy. 2014;100(1):36-40.

24. Jafarnezhadgero A, Alavi-Mehr SM, Granacher U. Effects of anti-pronation shoes on lower limb kinematics and kinetics in female runners with pronated feet: the role of physical fatigue. PLoS ONE. 2019;14(5):e0216818.

25. Jafarnezhadgero AA, Sorkhe E, Oliveira AS. Motion-control shoes help maintaining low loading rate levels during fatiguing running in pronated female runners. Gait Posture. 2019;73:65-70.

26. Jung D-Y, Kim M-H, Koh E-K, Kwon O-Y, Cynn H-S, Lee W-H. A comparison in the muscle activity of the abductor hallucis and the medial longitudinal arch angle during toe curl and short foot exercises. Phys Ther Sport. 2011;12(1):30-5.

27. Lee J, Yoon J, Cynn H. Foot exercise and taping in patients with patellofemoral pain and pronated foot. J Bodyw Mov Ther. 2017;21(1):216-22.

28. Wischnia B. Beach running. Runners World, July. 1982:76:48-9.

29. Pinnington HC, Lloyd DG, Besier TF, Dawson B. Kinematic and electromyography analysis of submaximal differences running on a firm surface compared with soft, dry sand. Eur J Appl Physiol. 2005;94(3):242-53.

30. van den Berg ME, Barr CJ, McLoughlin JV, Crotty M. Effect of walking on sand on gait kinematics in individuals with multiple sclerosis. Mult Scler Relat Disord. 2017;16:15-21.

31. Zamparo P, Perini R, Orizio C, Sacher M, Ferretti G. The energy cost of walking or running on sand. Eur J Appl Physiol. 1992;65(2):183-7.

32. Anderson M, Barnum M. Foundations of athletic training: Prevention, assessment, and management. Lippincott Williams \& Wilkins; 2021

33. Jung D-Y, Koh E-K, Kwon O-Y. Effect of foot orthoses and short-foot exercise on the cross-sectional area of the abductor hallucis muscle in subjects with pes planus: a randomized controlled trial 1. J Back Musculoskelet Rehabil. 2011;24(4):225-31.

34. Prentice WE. Rehabilitation techniques in sports medicine. McGraw-Hill Companies; 1999

35. Sulowska I, Oleksy Ł, Mika A, Bylina D, Sołtan J. The influence of plantar short foot muscle exercises on foot posture and fundamental movement patterns in long-distance runners, a non-randomized, non-blinded clinical trial. PLoS ONE. 2016;11(6):e0157917.

36. Sulowska I, Mika A, Oleksy Ł, Stolarczyk A. The influence of plantar short foot muscle exercises on the lower extremity muscle strength and power in proximal segments of the kinematic chain in long-distance runners. BioMed Res Int. 2019:2019:1-11.

37. Farahpour N, Jafarnezhadgero A, Allard P, Majlesi M. Muscle activity and kinetics of lower limbs during walking in pronated feet individuals with and without low back pain. J Electromyogr Kinesiol. 2018;39:35-41.

38. Bird AR, Bendrups AP, Payne CB. The effect of foot wedging on electromyographic activity in the erector spinae and gluteus medius muscles during walking. Gait Posture. 2003;18(2):81-91.

39. Semciw A, Neate R, Pizzari T. Running related gluteus medius function in health and injury: a systematic review with meta-analysis. J Electromyogr Kinesiol. 2016:30:98-110.

40. Barrett RS, Neal RJ, Roberts LJ. The dynamic loading response of surfaces encountered in beach running. J Sci Med Sport. 1998;1(1):1-11.

41. Yigit SS, Tuncel F. A comparison of the endurance training responses to road and sand running in high school and college students. J Strength Cond Res. 1998;12(2):79-81.

42. Tiberio D. The effect of excessive subtalar joint pronation on patellofemoral mechanics: a theoretical model. J Orthop Sports Phys Ther. 1987;9(4):160-5.

43. Farahpour N, Jafarnezhad A, Damavandi M, Bakhtiari A, Allard P. Gait ground reaction force characteristics of low back pain patients with pronated foot and able-bodied individuals with and without foot pronation. J Biomech. 2016:49(9):1705-10.

44. Redmond AC, Crosbie J, Ouvrier RA. Development and validation of a novel rating system for scoring standing foot posture: the Foot Posture Index. Clin Biomech. 2006;21(1):89-98.

45. Brody DM. Techniques in the evaluation and treatment of the injured runner. Orthop Clin North Am. 1982;13(3):541-58. 
46. McPoil TG, Cornwall MW. Relationship between three static angles of the rearfoot and the pattern of rearfoot motion during walking. J Orthop Sports Phys Ther. 1996;23(6):370-5.

47. Gijon-Nogueron G, Sanchez-Rodriguez R, Lopezosa-Reca E, Cervera-Marin JA, Martinez-Quintana R, Martinez-Nova A. Normal values of the Foot Posture Index in a young adult Spanish population: a cross-sectional study. J Am Podiatr Med Assoc. 2015;105(1):42-6.

48. Keenan A-M, Redmond AC, Horton M, Conaghan PG, Tennant A. The Foot Posture Index: Rasch analysis of a novel, foot-specific outcome measure. Arch Phys Med Rehabil. 2007;88(1):88-93.

49. Jakobsen MD, Sundstrup E, Andersen CH, Bandholm T, Thorborg K, Zebis MK, et al. Muscle activity during kneeextension strengthening exercise performed with elastic tubing and isotonic resistance. Int J Sports Phys Ther. 2012;7(6):606.

50. Sundstrup E, Jakobsen M, Andersen C, Bandholm T, Thorborg K, Zebis M, et al. Evaluation of elastic bands for lower extremity resistance training in adults with and without musculo-skeletal pain. Scand I Med Sci Sports. 2014;24(5):e353-9.

51. Sinclair J, Greenhalgh A, Edmundson CJ, Brooks D, Hobbs SJ. Gender differences in the kinetics and kinematics of distance running: implications for footwear design. Int J Sports Sci Eng. 2012;6(2):118-28.

52. Ferber R, Davis IM, Williams lii DS. Gender differences in lower extremity mechanics during running. Clin Biomech. 2003;18(4):350-7.

53. Durai DBJ, Shaju M. Effect of sand running training on speed among school boys. Int J Phys Educ Sports Health. 2019;6(3):117-22.

54. Anbarian $\mathrm{M}$, Esmaeili $\mathrm{H}$. Effects of running-induced fatigue on plantar pressure distribution in novice runners with different foot types. Gait Posture. 2016;48:52-6.

55. Chuckpaiwong B, Nunley JA, Mall NA, Queen RM. The effect of foot type on in-shoe plantar pressure during walking and running. Gait Posture. 2008;28(3):405-11.

56. Willems T, Witvrouw E, Delbaere K, De Cock A, De Clercq D. Relationship between gait biomechanics and inversion sprains: a prospective study of risk factors. Gait Posture. 2005;21(4):379-87.

57. Jafarnezhadgero AA, Shad MM, Majlesi M, Granacher U. A comparison of running kinetics in children with and without genu varus: A cross sectional study. PLoS ONE. 2017;12(9):e0185057.

58. Whittle MW. Gait analysis: an introduction. Butterworth-Heinemann; 2014.

59. Dugan SA, Bhat KP. Biomechanics and analysis of running gait. Phys Med Rehabil Clin. 2005;16(3):603-21.

60. Shorter AL, Rouse EJ. Ankle mechanical impedance during the stance phase of running. IEEE Trans Biomed Eng. 2019;67(6):1595-603.

61. Binnie MJ, Dawson B, Arnot MA, Pinnington H, Landers G, Peeling P. Effect of sand versus grass training surfaces during an 8-week pre-season conditioning programme in team sport athletes. J Sports Sci. 2014:32(11):1001-12.

62. Clarkson PM, Hubal MJ. Exercise-induced muscle damage in humans. Am J Phys Med Rehabil. 2002;81(11):S52-69.

\section{Publisher's Note}

Springer Nature remains neutral with regard to jurisdictional claims in published maps and institutional affiliations.

- fast, convenient online submission

- thorough peer review by experienced researchers in your field

- rapid publication on acceptance

- support for research data, including large and complex data types

- gold Open Access which fosters wider collaboration and increased citations

- maximum visibility for your research: over $100 \mathrm{M}$ website views per year

At BMC, research is always in progress.

Learn more biomedcentral.com/submissions 